\title{
CXCL1 and its receptor, CXCR2, mediate murine sickle cell vaso-occlusion during hemolytic transfusion reactions
}

\author{
Jung-Eun Jang, ${ }^{1}$ Eldad A. Hod, ${ }^{2}$ Steven L. Spitalnik, ${ }^{2}$ and Paul S. Frenette ${ }^{1,3}$ \\ 1Department of Medicine, Mount Sinai School of Medicine, New York, New York, USA. ²Department of Pathology and Cell Biology, Columbia University \\ College of Physicians and Surgeons, New York, New York, USA. ${ }^{3}$ Ruth L. and David S. Gottesman Institute for Stem Cell and Regenerative Medicine Research, \\ Albert Einstein College of Medicine, New York, New York, USA.
}

\begin{abstract}
Hemolytic transfusion reactions (HTRs) can produce serious and potentially life-threatening complications in sickle cell disease (SCD) patients; however, the mechanisms underlying these complications remain undetermined. We established a model of alloimmune, IgG-mediated HTRs in a well-characterized humanized murine model of SCD. HTRs induced acute vaso-occlusive crisis (VOC), resulting in shortened survival of SCD mice. Acute VOC was associated with elevated circulating inflammatory chemokine levels, including striking elevation of the levels of the neutrophil chemoattractant CXCL1. Recombinant CXCL1 administration was sufficient to induce acute VOC in SCD mice, characterized by leukocyte recruitment in venules, capture of circulating red blood cells, reduction of venular flow, and shortened survival. In contrast, blockade of the CXCL1 receptor, CXCR2, prevented HTR-elicited acute VOC and prolonged survival in SCD mice. These results indicate that CXCL1 is a key inflammatory mediator of acute VOC in SCD mice. Targeted inhibition of CXCL1 and/or CXCR2 may therefore represent a new therapeutic approach for acute VOC in SCD patients.
\end{abstract}

\section{Introduction}

Sickle cell disease (SCD) is a genetic disorder caused by a single mutation in $\beta$-globin (1). Hemoglobin $\mathrm{S}\left(\mathrm{Hb}^{\mathrm{s}}\right)$, containing the mutated $\beta$-globin, causes changes in rbc shape, stiffness, and adhesiveness, thereby altering rheological properties and inducing hemolysis and vaso-occlusion (VOC) (1-3). Although rbc transfusions correct anemia, improve rheological properties, and increase oxygen-carrying capacity in SCD patients, they can produce potentially dangerous immunological responses as a result of repeated exposure to allogeneic blood group antigens (4-7).

Delayed hemolytic transfusion reactions (HTRs) are typically IgG-mediated systemic responses caused by blood group antigen incompatibility. IgG-mediated HTRs involve complement activation, phagocytosis, cytokine production, and various cellular responses (8-11). In SCD, HTRs can precipitate acute VOC or hyperhemolysis syndrome, resulting in significant morbidity (5-7). However, the mechanisms mediating the severe complications of HTRs in SCD patients are unknown, and no specific therapy is available. Because inflammatory cytokines and chemokines are important in the pathogenesis of acute and delayed HTRs and in SCD crises $(10,12-14)$, they may be involved in the serious and specific clinical manifestations of HTRs found in SCD patients.

To date, acute VOC models have relied on pharmacological (e.g., cytokines; refs. 15, 16) or physical (e.g., surgery, hypoxia/ reoxygenation; refs. $15,17-19)$ interventions that may or may not faithfully reflect human SCD crises. While investigating a murine HTR model, we found that the transfusion reaction to incompatible blood was, as in humans, sufficient to induce lethal VOC in SCD mice. Thus, this approach can provide an opportunity

Authorship note: Jung-Eun Jang and Eldad A. Hod contributed equally to this work. Conflict of interest: The authors have declared that no conflict of interest exists. Citation for this article: J Clin Invest. 2011;121(4):1397-1401. doi:10.1172/JCI45336. to gain insights about key endogenously produced mediators of VOC. Here, we have identified the chemokine CXCL1 as a critical mediator inducing severe VOC in humanized SCD mice.

\section{Results and Discussion}

Alloimmune, IgG-mediated HTR model in SCD mice. Human glycophorin A-transgenic (hGPA-Tg) mice on an FVB background and control wild-type FVB mice were used as donors of incompatible and compatible rbcs, respectively. HTRs were induced by passive immunization with a monoclonal IgG anti-hGPA antibody, as described previously $(9,20)$; thus, fluorescently labeled rbcs from hGPA-Tg or FVB mice were transfused into humanized SCD mice followed by passive immunization with anti-hGPA antibody (Supplemental Figure 1A; supplemental material available online with this article; doi:10.1172/JCI45336DS1). The survival of incompatible hGPA-Tg rbcs was markedly reduced $(20 \% \pm 5 \% ; n=6)$, whereas the survival of FVB rbcs was largely preserved $(95 \% \pm 4 \% ; n=7)$, at 5 hours after transfusion in passively immunized SCD mice, as determined by serial flow cytometric analysis (Supplemental Figure 1, B and C). The reduction of transfused rbcs was specific because there were no significant differences in the number of wbcs (i.e., leukocytes), platelets, endogenous rbcs, or reticulocytes between the 2 groups of recipients 2 hours after HTRs (Table 1 ).

IgG-mediated HTRs induce acute VOC. We then evaluated the effect of HTRs on the microvasculature of live SCD mice using intravital microscopy. We found a significant reduction in the mean blood flow rate, a surrogate measure of VOC, at 1 hour after transfusion of hGPA-Tg compared with FVB rbcs $(262 \pm 19$ vs. $335 \pm 57 \mathrm{nl} / \mathrm{s}$, $P<0.01$; Figure 1A). This difference was not attributable to vessel size because the average venular diameter was nearly identical between the 2 groups (Supplemental Table 1). The mean centerline rbc velocity $\left(V_{r b c}\right)$ and wall shear rate were also significantly reduced by 2 hours after transfusion of hGPA-Tg compared with FVB rbcs $(P<0.05$ and $P<0.001$, respectively; Supplemental Table 1). Since acute VOC is 


\section{brief report}

Table 1

Effect of IgG-mediated HTRs on peripheral blood counts in SCD mice

\begin{tabular}{|c|c|c|c|c|c|c|}
\hline $\begin{array}{l}\text { Transfusion } \\
\text { (rbcs) }\end{array}$ & $\begin{array}{c}\text { wbcs } \\
\left(10^{3} / \mu l\right)\end{array}$ & $\begin{array}{c}\text { rbcs } \\
\left(10^{6} / \mu l\right)\end{array}$ & $\begin{array}{l}\text { Hemoglobin } \\
(\mathrm{g} / \mathrm{dl})\end{array}$ & $\begin{array}{c}\text { Hematocrit } \\
(\%)\end{array}$ & $\begin{array}{l}\text { Platelets } \\
\left(10^{3} / \mu \mathrm{I}\right)\end{array}$ & $\begin{array}{c}\text { Reticulocytes } \\
\left(10^{9} / I\right)\end{array}$ \\
\hline & & & & & & \\
\hline
\end{tabular}

Data are mean $\pm \operatorname{SEM}(n=4$ per group). thelium by 1.4 -fold $(2,396 \pm 168$ vs. $1,667 \pm 73$ adherent wbcs $\left./ \mathrm{mm}^{2} ; P<0.001\right)$ and rbc-wbc interactions by 5.3 -fold $(1.0 \pm 0.2$ vs. $0.2 \pm 0.1$ interactions/minute; $P<0.05)$ at $91-120$ minutes after HTR initiation, leading to acute VOC in postcapillary venules by 2 hours after HTR initiation (Figure 1, B-D, and Supplemental Videos 1 and 2). After the end of the recording period, mice were monitored for up to 9 hours after anti-hGPA antibody infusion to assess the effect of HTRs on mortality in induced by interactions between sickle rbcs and adherent wbcs in the microvasculature of TNF- $\alpha$-treated SCD mice $(15,16)$, we also evaluated wbc recruitment and rbc-wbc interactions in SCD mice with HTRs (without prior proinflammatory cytokine treatment). Compared with compatible transfusions with FVB rbcs, the transfusion of incompatible hGPA-Tg rbcs, followed by passive immunization with anti-hGPA antibody, significantly increased wbc adhesion to endo-
SCD mice. The survival of passively immunized SCD mice transfused with hGPA-Tg compared with FVB rbcs was significantly reduced $(P<0.05$, log-rank test; Figure 1E). Thus, the transfusion of incompatible rbcs is sufficient to induce typical VOC in SCD mice.

In previous studies, transfusion of incompatible rbcs into passively alloimmunized non-SCD mice induced cytokine storm (10). To determine whether similar effects occur in SCD mice with
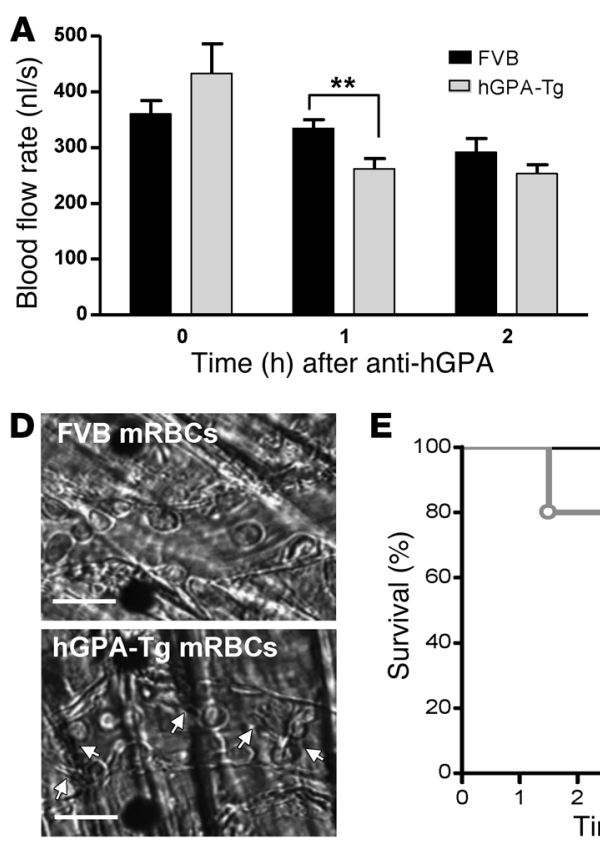

$E$

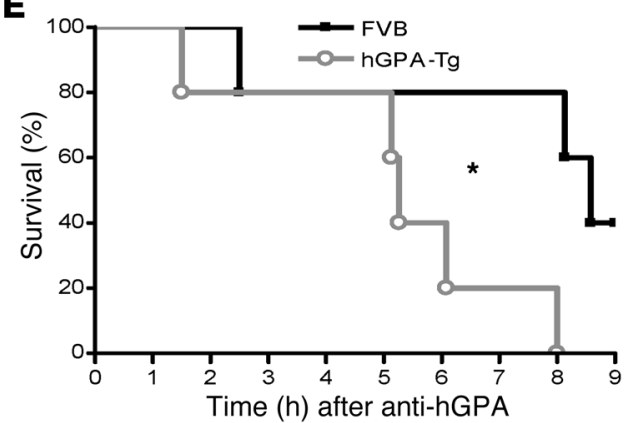

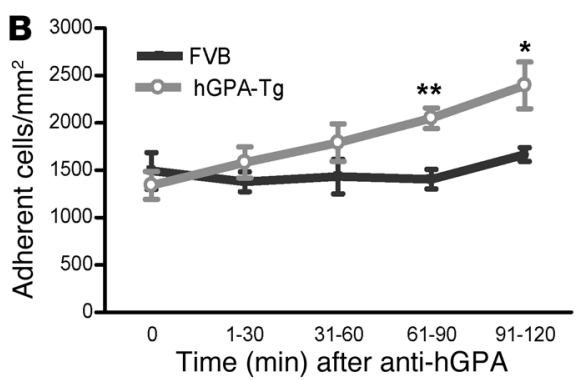

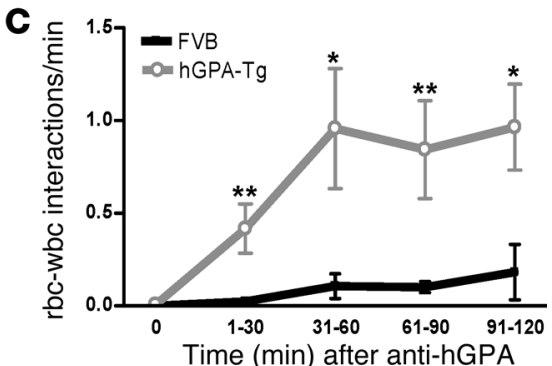

F
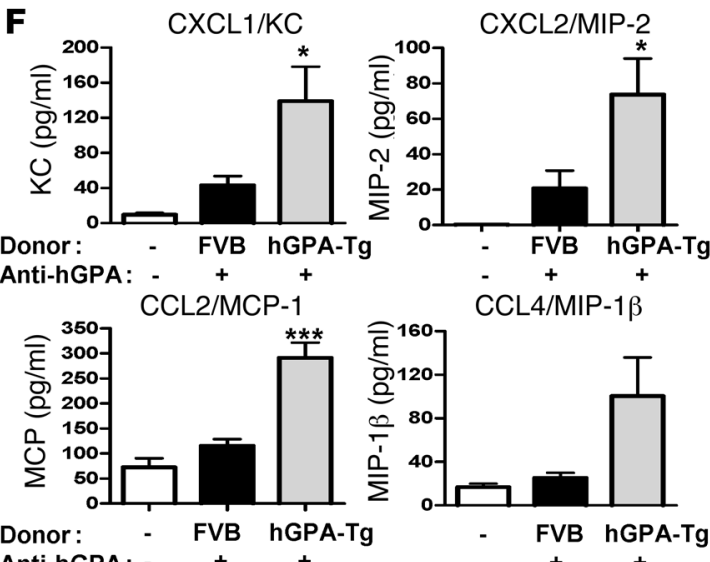

Anti-hGPA: -
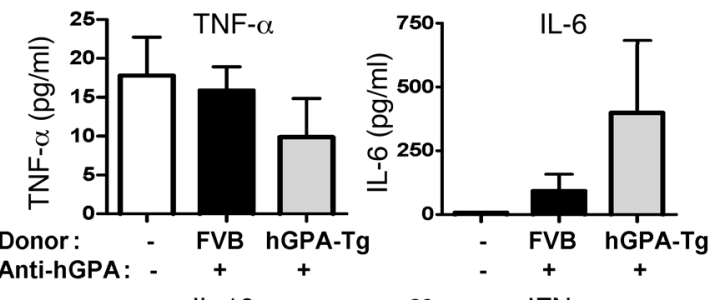

HTRs induce acute VOC and cytokine production in SCD mice. SCD mice ( $n=7$ per group) were transfused with compatible wild-type FVB rbcs or incompatible hGPA-Tg rbcs, followed by passive immunization with anti-hGPA antibody. (A) Blood flow rate. (B) Adherent wbcs. (C) Number of circulating sickle rbc-adherent wbc interactions. (D) Representative images 91-120 minutes after anti-hGPA antibody infusion. Arrows denote sickle rbc-adherent wbc interactions. Scale bars: $20 \mu \mathrm{m}$. (E) Kaplan-Meier survival curves $(P=0.03$, log-rank test). (F) Plasma cytokine concentration 2 hours after passive immunization. Control parental SCD mice were not infused with anti-hGPA antibody or transfused with rbcs. ${ }^{*} P<0.05$, ${ }^{* *} P<0.01,{ }^{* * *} P<0.001$ versus FVB.
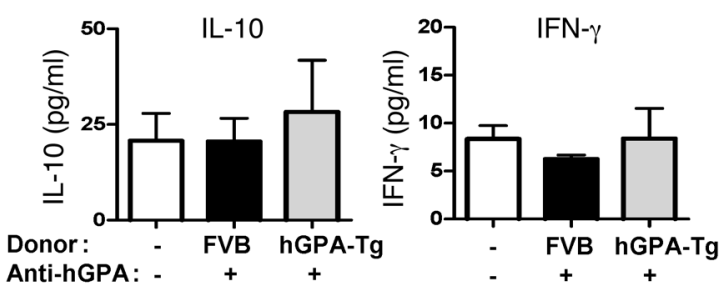


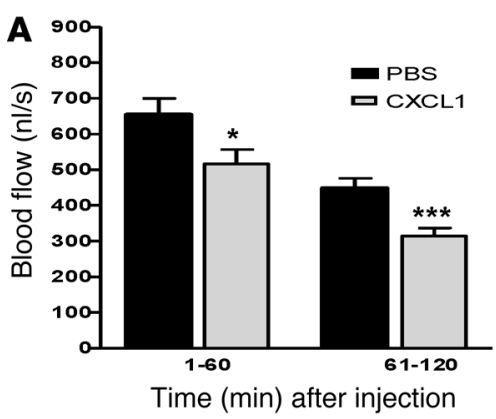

D

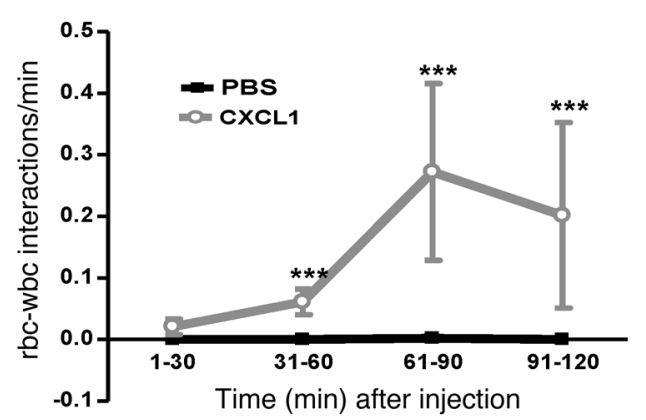

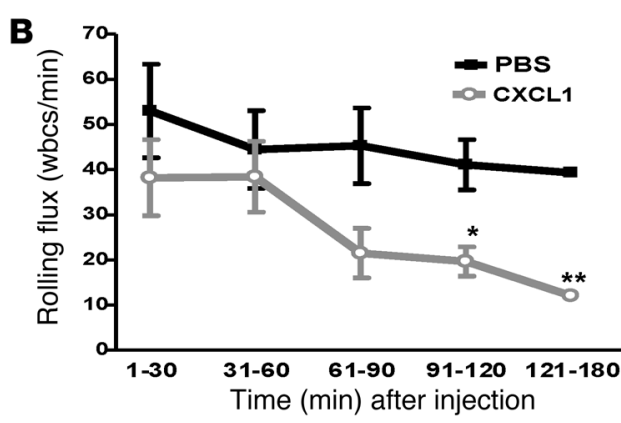

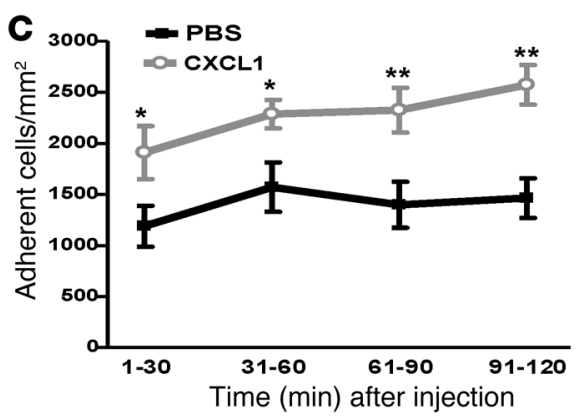

E
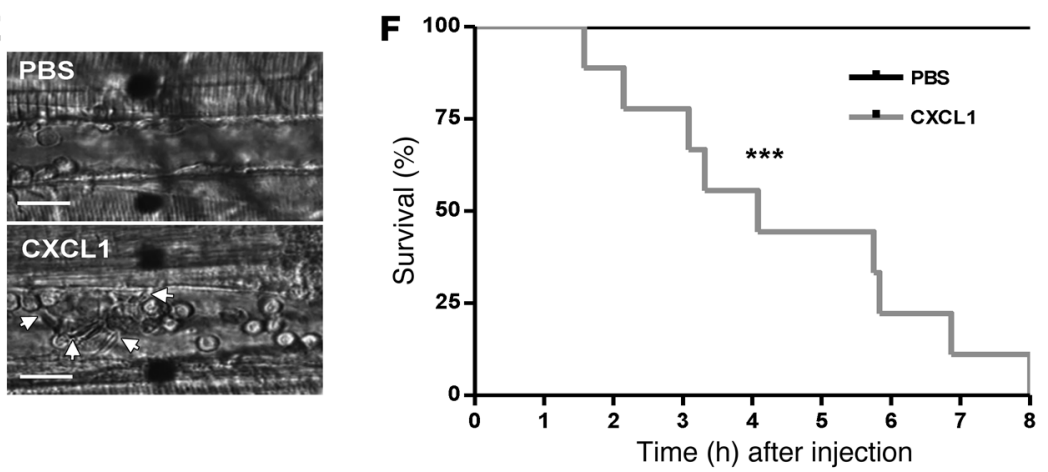

Figure 2

CXCL1 infusion induces acute VOC. CXCL1 (300 pg) or PBS control was intravenously injected into SCD mice ( $n=10$ or 7 per group). (A) Blood flow. (B) Number of rolling wbcs. (C) Adherent wbcs. (D) Number of circulating sickle rbc-adherent wbc interactions. (E) Real-time intravital microscopy 91-120 minutes after CXCL1 infusion. Arrows denote sickle rbc-adherent wbc interactions. Scale bars: $20 \mu \mathrm{m}$. (F) Kaplan-Meier survival curves $(P<0.001$, log-rank test $) .{ }^{*} P<0.05,{ }^{* *} P<0.01,{ }^{* \star} P<0.001$ versus PBS.

HTRs and associated acute VOC, levels of circulating inflammatory cytokines and chemokines were measured. Plasma levels of CXCL1, CXCL2, and CCL2 were significantly increased $(P<0.05$, $P<0.05$, and $P<0.001$, respectively) in passively immunized SCD mice 2 hours after transfusion of hGPA-Tg rbcs, compared with SCD mice transfused with FVB rbcs and with parental steady-state SCD mice (Figure 1F). In contrast, plasma levels of CCL4, TNF- $\alpha$, IL-6, IL-10, and IFN- $\gamma$ did not change significantly (Figure 1F). The temporal association of elevated chemokine levels with heterotypic rbc-wbc interactions following incompatible rbc transfusion suggests that endogenously produced chemokines may be involved in the pathophysiology of acute VOC in this mouse model.

CXCL1 triggers acute VOC. Because neutrophils play a critical role in the heterotypic interactions that cause VOC in SCD mice $(15,16,21)$, we hypothesized that the high levels of neutrophil chemoattractant mediate HTR-induced acute VOC. To test this hypothesis, SCD mice were injected with the recombinant chemokines that were found to be elevated in blood: CXCL1, CXCL2, and CCL2 (Figure 1F). Exogenous CXCL1 significantly reduced blood flow, mean centerline $V_{r b c}$, and wall shear rate compared with PBS control infusion in postcapillary venules of SCD mice by 2 hours after administration (Figure 2A and Supplemental Table 2). Administration of CXCL1 significantly altered wbc recruitment compared with PBS by reducing the number of rolling wbcs up to 2 -fold ( $20 \pm 3$ vs. $41 \pm 6$ rolling wbcs/minute; $P<0.05$; Figure $2 \mathrm{~B}$ ), but increasing wbc adhesion to the endothelium $\left(2,571 \pm 193\right.$ versus $1,464 \pm 194$ adherent $\mathrm{wbcs} / \mathrm{mm}^{2} ; P<0.01$; Figure $2 \mathrm{C}$ ). Recruited wbcs frequently interacted with circulating sickle rbcs, resulting in intermittent acute VOC in the postcapillary venules of SCD mice treated with CXCL1 compared with PBS-infused con- trols (Figure 2, D and E, and Supplemental Videos 3 and 4). CXCL1induced acute VOC in SCD mice led to shorter survival times compared with control PBS-infused SCD mice $(P<0.001$, log-rank test; Figure $2 \mathrm{~F}$ ), and the median survival time of CXCL1-treated SCD mice (5 hours) was very similar to that of SCD mice with HTRs. In contrast to CXCL1-infused SCD mice, administration of exogenous CXCL2 or CCL2 had no significant effect in SCD mice on blood flow rate, $w b c$ recruitment, rbc-wbc interactions, or survival time (Supplemental Figure 2, A-D, and Supplemental Table 3). Taken together, these findings suggest that increased CXCL1 levels alone are sufficient to initiate $\mathrm{wbc}$ recruitment and induce acute VOC in SCD mice and that endogenously released CXCL1 may play an important role in HTR-induced acute VOC.

CXCR2 inbibition prevents HTR-induced acute VOC in SCD mice. CXCR2, the only known receptor for CXCL1, mediates neutrophil chemotaxis in response to many types of infections $(22,23)$. Thus, we investigated whether pharmacological blockade of CXCR2 prevents acute VOC in this model of HTR in SCD mice. The CXCR2 antagonist SB225002 did not affect clearance of transfused incompatible rbcs compared with DMSO controls 2 hours after transfusion $(P=0.54$; Figure $3 \mathrm{~A})$. However, pretreatment of SCD mice with the CXCR2 antagonist improved by approximately 2 -fold both blood flow (381 \pm 39 vs. $211 \pm 18 \mathrm{nl} / \mathrm{s} ; P<0.01)$ and mean centerline $V_{r b c}(2.0 \pm 0.2$ vs. $1.1 \pm 0.1 \mathrm{~mm} / \mathrm{s} ; P<0.01)$ and decreased wbc adhesion to endothelium approximately 2 -fold $\left(1,160 \pm 159\right.$ vs. $2,250 \pm 114$ adherent wbcs $\left./ \mathrm{mm}^{2} ; P<0.01\right)$ between 61 and 120 minutes after HTR induction compared with controls (Figure 3, B and C, and Supplemental Table 4). Heterotypic rbc-wbc interactions were significantly reduced; moreover, 

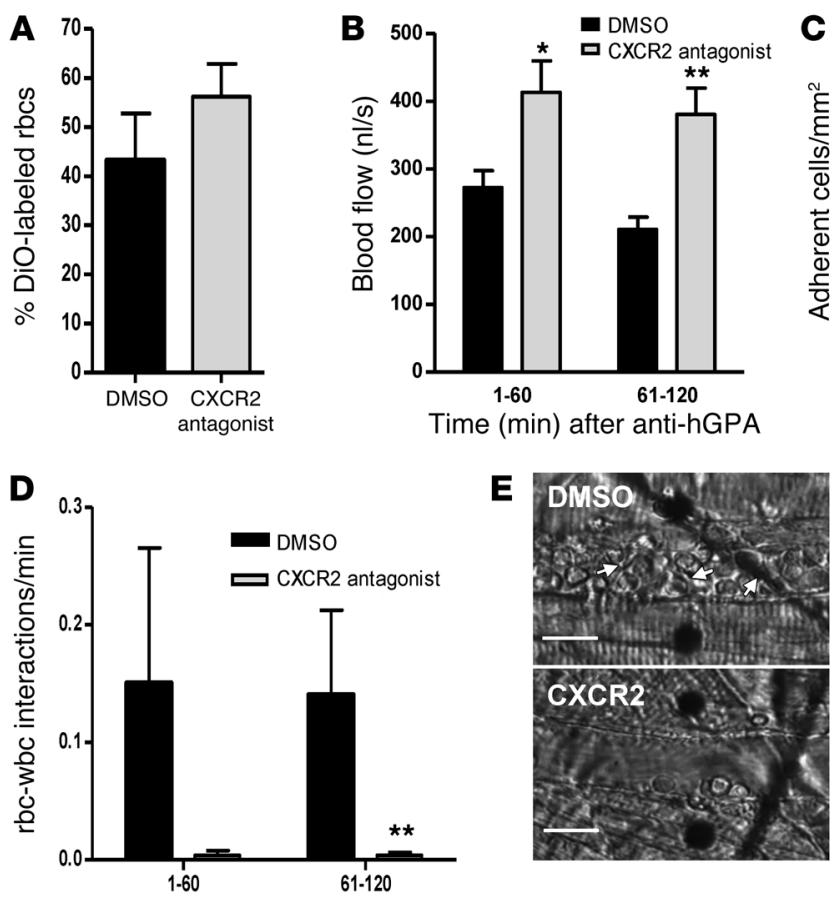

Time (min) after anti-hGPA

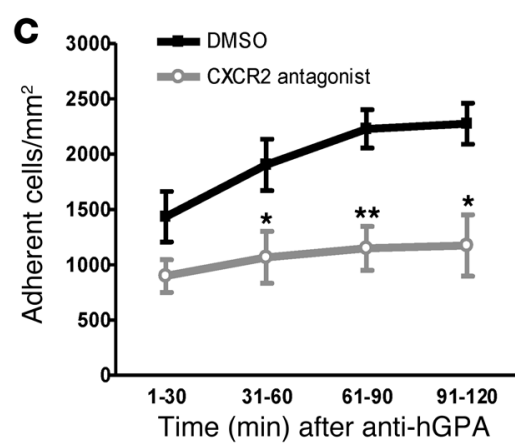

$\mathbf{F}$

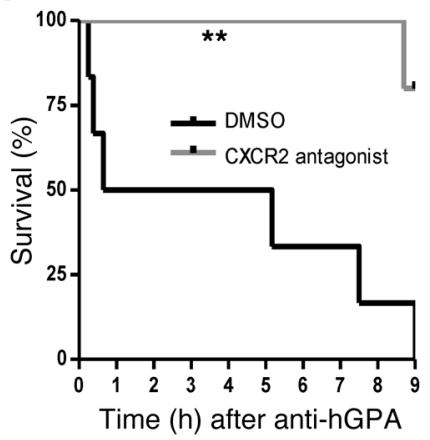

Figure 3

CXCR2 blockade prevents HTR-induced acute VOC in SCD mice. At 30 minutes before passive immunization with anti-hGPA antibodies for HTR induction, SCD mice ( $n=5$ or 6 per group) were injected intravenously with CXCR2 antagonist SB225002 (4.5 mg/kg) or DMSO vehicle control. (A) Survival of transfused fluorescently labeled rbcs 2 hours after HTR induction. (B) Blood flow. (C) Adherent wbcs. (D) Number of circulating sickle rbc-adherent wbc interactions. (E) Real-time intravital microscopy 91-120 minutes after HTR induction. Arrows denote sickle rbc-adherent wbc interactions. Scale bars: $20 \mu \mathrm{m}$. (F) Kaplan-Meier survival curves $\left(P<0.01\right.$, log-rank test). ${ }^{\star} P<0.05,{ }^{\star \star} P<0.01$ versus DMSO.

HTR-induced acute VOC was prevented (Figure 3, D and E, and Supplemental Videos 5 and 6). Furthermore, pharmacological blockade of CXCR2 significantly decreased HTR-induced mortality in SCD mice compared with controls $(P<0.01$, log-rank test; Figure 3F). Taken together, these results strongly suggest that blockade of CXCR2 function may be beneficial in the treatment of HTR-induced acute VOC.

In conclusion, we demonstrated that IgG-mediated HTR induced acute VOC in SCD mice, that CXCL1 was a critical inflammatory mediator of acute VOC, and that targeted blockade of its receptor, CXCR2, prevented the adverse clinical effects of HTRs in this mouse SCD model. These results suggest a possible pharmacological approach for treating this serious complication of transfusion therapy in SCD patients.

\section{Methods}

Animals. Berkeley SCD mice ( $\left.\operatorname{Tg}\left[\mathrm{Hu}-\mathrm{miniLCR} \alpha 1^{\mathrm{G}} \gamma^{\mathrm{A}} \gamma \delta \beta^{\mathrm{S}}\right] H b a^{-/-} \mathrm{Hb} b^{-/-}\right)$ were described previously (20). Bone marrow nucleated cells from Berkeley SCD mice were transplanted into lethally irradiated C57BL/ 6 recipients to generate age- and gender-matched genetically identical cohorts of SCD mice. Fully chimeric male SCD mice (expressing >97\% human globin, including $\beta S$ ) were studied by intravital microscopy 3-5 months after bone marrow transplantation (15). hGPA-Tg mice expressing the GYPA gene were maintained as described previously (9). All experimental procedures were approved by the Animal Care and Use Committees of Mount Sinai School of Medicine, Columbia University Medical Center, and Albert Einstein College of Medicine.
Model of alloimmune, IgG-mediated HTR. Washed, buffy coat-depleted rbcs from wild-type FVB/NJ and hGPA-Tg mice were labeled with chloromethylbenzamido 1,1'-dioctadecyl-3,3,3',3'-tetramethylindocarbocyanine perchlorate ( $\mathrm{DiO}$ ) (Invitrogen) and resuspended to a $40 \%$ hematocrit in PBS (10). We intravenously transfused $50 \mu \mathrm{l}$ of DiO-labeled hGPA-Tg or wild-type FVB/NJ rbcs into SCD mice, which were then passively immunized with $10 \mu \mathrm{g}$ purified 6A7 IgG1 anti-hGPA antibody $(9,10)$. Survival of rbcs was assessed by quantifying the percentage of DiO-labeled rbcs in the circulation by flow cytometry (FACSCalibur; BD) at defined time points. Peripheral blood counts were determined using an ADVIA 120 Hematology Analyzer System (Bayer).

Intravital microscopy. The cremasteric muscle was prepared, and brightfield intravital microscopy performed using video recordings as described previously $(15,16)$. Recording of postcapillary and collecting venules was performed for 2 hours after HTR induction (with time 0 defined as surgical sectioning of the cremaster muscle immediately prior to injection of anti-hGPA antibody), and each venule was recorded continuously for 2 minutes. To evaluate the role of CXCL1 in SCD mice, $300 \mathrm{pg}$ recombinant murine CXCL1 (R\&D Systems) or control PBS was intravenously injected after surgical preparation, and images were recorded for 2 hours. In some experiments, SCD mice were intravenously injected with CXCR2 antagonist SB225002 (4 mg/kg; TOCRIS bioscience) or DMSO vehicle 30 minutes before HTR induction, and images were then recorded for 2 hours. Venules were visualized as described previously $(15,16)$. Mouse survival time was defined as the time interval from HTR induction to death.

Hemodynamic measurements and image analyses for bright-field intravital microscopy. Venular diameter was measured with a video caliper. $V_{r b c}$ was 
measured for each venule in real time using an optical Doppler velocimeter at Texas A\&M University. Wall shear rate and blood flow rate were calculated as described previously $(15,16)$. Adherent wbcs were defined as those remaining stationary for at least 30 seconds over a $100-\mu \mathrm{m}$ venular segment. rbcs were identified by their size and shape (discoid and sickle-shaped cells). An interaction between rbcs and adherent wbcs was defined as the arrest of an $\mathrm{rbc}$ on an adherent $\mathrm{wbc}$ for more than 2 video frames (>0.07 seconds; refs. 15, 16).

Cytokine analysis. Plasma separated from blood samples obtained by cardiac puncture 2 hours after HTR induction was used to quantify cytokines using Cytometric Bead Array Mouse Flex Kits (BD Biosciences). CXCL2 was quantified using mouse CXCL2 immunoassay kits (R\&D Systems).

Statistics. All data are presented as mean \pm SEM and analyzed using unpaired, 2-tailed Student's $t$ test or nonparametric Mann-Whitney $U$ test as appropriate. A $P$ value less than 0.05 was considered statistically significant.

1. Steinberg MH. Management of sickle cell disease. NEngl J Med. 1999;340(13):1021-1030.

2. Frenette PS, Atweh GF. Sickle cell disease: old discoveries, new concepts, and future promise. J Clin Invest. 2007;117(4):850-858.

3. Morris CR. Mechanisms of vasculopathy in sickle cell disease and thalassemia. Hematology Am Soc Hematol Educ Program. 2008:177-185.

4. Wayne AS, Kevy SV, Nathan DG. Transfusion management of sickle cell disease. Blood. 1993; 81(5):1109-1123

5. Cox JV, Steane E, Cunningham G, Frenkel EP. Risk of alloimmunization and delayed hemolytic transfusion reactions in patients with sickle cell disease. Arch Intern Med. 1988;148(11):2485-2489.

6. Vichinsky EP. Current issues with blood transfusions in sickle cell disease. Semin Hematol. 2001; 38(1 suppl 1):14-22.

7. Diamond WJ, Brown FL Jr, Bitterman P, Klein HG, Davey RJ, Winslow RM. Delayed hemolytic transfusion reaction presenting as sickle-cell crisis. Ann Intern Med. 1980;93(2):231-234.

8. Davenport RD. Pathophysiology of hemolytic transfusion reactions. Semin Hematol. 2005;42(3):165-168.

9. Schirmer DA, et al. Mouse models of IgG- and IgMmediated hemolysis. Blood. 2007;109(7):3099-3107.

\section{Acknowledgments}

We thank Barry Coller for providing access to the ADVIA automated cell counter. This work was supported by NIH grants R01HL69438, RC1HL099545, and R01HL097700 (to P.S. Frenette), R01HL098014 and U01HD064827 (to S.L. Spitalnik), and K08 HL103756 (to E.A. Hod); by a Louis V. Gerstner Scholars Award (to E.A. Hod); and by a Predoctoral Fellowship from the American Heart Association (to J.-E. Jang). P.S. Frenette is an Established Investigator of the American Heart Association.

Received for publication October 6, 2010, and accepted in revised form January 12, 2011.

Address correspondence to: Paul S. Frenette, Gottesman Institute for Stem Cell and Regenerative Medicine, Price Center, 1301 Morris Park Ave., New York, New York 10461, USA. Phone: 718.678.1255; Fax: 718.678.1018; E-mail: paul.frenette@einstein.yu.edu.

10. Hod EA, et al. Cytokine storm in a mouse model of IgG-mediated hemolytic transfusion reactions. Blood. 2008;112(3):891-894.

11. Meyer D, et al. FcgammaRIII (CD16)-deficient mice show IgG isotype-dependent protection to experimental autoimmune hemolytic anemia. Blood. 1998;92(11):3997-4002.

12. Davenport RD, Burdick M, Moore SA, Kunkel SL. Cytokine production in IgG-mediated red cell incompatibility. Transfusion. 1993;33(1):19-24.

13. Rakic S. [New findings on the physiopathology of acute hemolytic transfusion reactions]. Med Pregl. 1999;52(1-2):19-24.

14. Goncalves MS, et al. Interleukin 8 as a vaso-occlusive marker in Brazilian patients with sickle cell disease. Braz J Med Biol Res. 2001;34(10):1309-1313.

15. Turhan A, Weiss LA, Mohandas N, Coller BS, Frenette PS. Primary role for adherent leukocytes in sickle cell vascular occlusion: a new paradigm. Proc Natl Acad Sci U S A. 2002;99(5):3047-3051.

16. Chang J, Shi PA, Chiang EY, Frenette PS. Intravenous immunoglobulins reverse acute vaso-occlusive crises in sickle cell mice through rapid inhibition of neutrophil adhesion. Blood. 2008;111(2):915-923.

17. Kaul DK, Hebbel RP. Hypoxia/reoxygenation causes inflammatory response in transgenic sickle mice but not in normal mice. J Clin Invest. 2000; 106(3):411-420.

18. Kalambur VS, et al. Microvascular blood flow and stasis in transgenic sickle mice: utility of a dorsal skin fold chamber for intravital microscopy. Am J Hematol. 2004;77(2):117-125.

19. Belcher JD, et al. Critical role of endothelial cell activation in hypoxia-induced vasoocclusion in transgenic sickle mice. Am J Physiol Heart Circ Physiol. 2005;288(6):H2715-H2725.

20. Paszty C, et al. Transgenic knockout mice with exclusively human sickle hemoglobin and sickle cell disease. Science. 1997;278(5339):876-878.

21. Hidalgo A, Chang J, Jang JE, Peired AJ, Chiang EY, Frenette PS. Heterotypic interactions enabled by polarized neutrophil microdomains mediate thromboinflammatory injury. Nat Med. 2009; 15(4):384-391.

22. Belperio JA, et al. Critical role for CXCR2 and CXCR2 ligands during the pathogenesis of ventilator-induced lung injury. J Clin Invest. 2002; 110(11):1703-1716

23. Chintakuntlawar AV, Chodosh J. Chemokine CXCL1/KC and its receptor CXCR2 are responsible for neutrophil chemotaxis in adenoviral keratitis. J Interferon Cytokine Res. 2009;29(10):657-666. 